\title{
Gyroscope System Identification using an Impulse Response RLS Algorithm
}

\author{
Eyad Turki \\ Mechanical Engineering Department \\ Jordan Univ. of Science \& Tech \\ P.O.Box 3030 \\ Irbid, Jordan \\ Eyad_17@yahoo.com
}

\author{
Tarek A. Tutunji \\ Mechatronics Engineering Department \\ Philadelphia University \\ PO Box 1 \\ 19392 - Jordan \\ ttutunji@yahoo.com
}

\author{
Mohammad Molhim \\ Mechanical Engr Department \\ Jordan Univ. of Science \& Tech \\ P.O.Box 3030 \\ Irbid, Jordan \\ molhim@just.edu.jo
}

\begin{abstract}
A recursive identification algorithm is used to identify a gyroscope system using impulse response data. The algorithm is based on an Auto Regressive Moving Average model with a steepest descent method. Impulse voltage input is used to excite the system and the angular velocity output is measured. The angular velocity output is dependent on the voltage and current inputs and affected by the system constants: motor inductance and mechanical constant, gear ratio and efficiency, moment of inertia, and load. The transfer function is constructed from the identified model using the Z-transform. Then, the input voltage is varied and applied to both: the identified model and the physical system. Experimental results using Labview with Data Acquisition Card (DAQ) are presented. Results show that the recursive identification algorithm is able to identify the systems with minimal error.
\end{abstract}

\section{INTRODUCTION}

System Identification is the field of modelling dynamic systems from experimental data (i.e. input/output patterns). The goal is to approximate the 'unknown' black box with a linear regression model that uses the available input/output data.

System identification gained much interest since the early 1990's [1]. Recently, researchers developed the system identification methods and applied them to many engineering systems. Recursive least squares method was used to estimate the unknown parameters of a DC motor system by matching input-output behaviour of the physical system [2] and [3]. Impulse response data was used as patterns in a recursive gradient algorithm to identify the transfer function of a DC motor [4].

Structured recurrent neural network were used to identify physical relevant parameters and nonlinear characteristics of a nonlinear two-mass system with friction and backlash [5]. A least square derived algorithm was used to estimate the rotor resistance, self-inductance of the rotor winding, as well as the stator leakage inductance of a three phase induction machine [6].

Recursive prediction error method based on the Autoregressive Moving Average model was used to identify the transfer function of a CNC milling machine in order to apply a combined self-tuning adaptive control and crosscoupling control to retrofit the machine with DC motors instead of stepper motors [7]. Robotics is another application area for system identification [8] and [9].

In this paper, a recursive least square (RLS) algorithm was applied to identify a gyroscope system. An input impulse voltage was used to excite the system and the angular velocity was measured. This input-output data pattern was used in an Auto Regressive Moving Average (ARMA) model. RLS algorithm based on steepest descent was used to identify the ARMA parameters and ultimately the transfer function. Then, the input voltage was changed relative to the gyroscope motor and the output was predicted accurately. More details of this work were presented in [10].

The gyroscope system is highly nonlinear. The gyroscope is used in many navigation systems such as airplanes and ships. The relationship between the voltage input and angular velocity output is somewhat complicated. The identification of such systems is of interest since it can predict the output and contribute to its control.

This paper is divided as follows: section 2 gives the mathematical background of the recursive algorithm used to identify the mechatronic systems tested. Section 3 gives the gyroscope modelling. Section 4 presents the experimental results of the gyroscope. Finally, the conclusions are summarized in section 5 .

\section{RECURSIVE IDENTIFICATION}

The model structure used to identify the system dynamics for a single-input-single-output is given below [1]

$$
y(k)=\Phi(k)^{T} \theta+\varepsilon(k)
$$

where $y(k)$ is the output at time $\mathrm{k}, \Phi^{T}(k)$ is the $\theta$ dimensional vector whose elements are the delayed input, $u(k-d l)$ and output components,

$$
\phi^{T}(k)=\left[\begin{array}{llllll}
-y(k-1) & \ldots & -y(k-n) & u(k-1) & \ldots & u(k-m)
\end{array}\right]
$$

and $\theta$ is the $\theta$-dimensional parameter vector and $\varepsilon(k)$ is the equation error.

$$
\theta=\left[\begin{array}{llllll}
a_{1} & \ldots & a_{n} & b_{1} & \ldots & b_{m}
\end{array}\right]
$$

The ARMA model can be presented as

$$
A y(k)=B u(k)+\varepsilon(k)
$$

where $A=1+a_{1} Z^{-1}+a_{2} Z^{-2}+\ldots+a_{n} Z^{-n}$

and

$$
B=b_{1} Z^{-1}+b_{2} Z^{-2}+\ldots+b_{m} Z^{-m}
$$

Simple least square estimate of the parameter vector $\theta$ is 
$\hat{\theta}=\left[\sum_{t=1}^{N} \phi(k) \phi^{T}(k)\right]^{-1}\left[\sum_{t=1}^{N} \phi(k) y(k)\right]$

Recursive Least Square (RLS) gives the following equations

$$
\begin{aligned}
& \hat{\theta}(k)=\hat{\theta}(k-1)+Q(k) \mathcal{\varepsilon}(k) \\
& \varepsilon(k)=y(k)-\varphi^{T}(k) \hat{\theta}(k-1) \\
& Q(k)=P(k-1) \varphi(k) /\left[\lambda+\varphi^{T}(k) P(k-1) \varphi(k)\right\}
\end{aligned}
$$

where $P(k)$ is a diagonal positive definite matrix and $\lambda$ is the forgetting factor. In an ARMA model, the system to be identified is assumed to have the following linear model

$$
\hat{y}(k)=\sum_{j=1}^{n} a(j) y(k-j)+\sum_{i=0}^{m} b(i) u(k-i)
$$

where $u(k), y(k)$ and $\hat{y}(k)$ are the input, the original system output, and the predicted model output of the $\mathrm{k}^{\text {th }}$ sample.

The goal is to find a linear system model that gives output, $\hat{y}$, equal to real output $\mathrm{y}$. The error between the actual and modelled output is $\quad$ error $=\hat{y}-y$

Input-output patterns $(u, y)$ are available. They are used in the above equations to calculate $\hat{y}$. The parameters $a(i)$ and $b(j)$ are updated to minimize the least square error as shown in figure 1 .

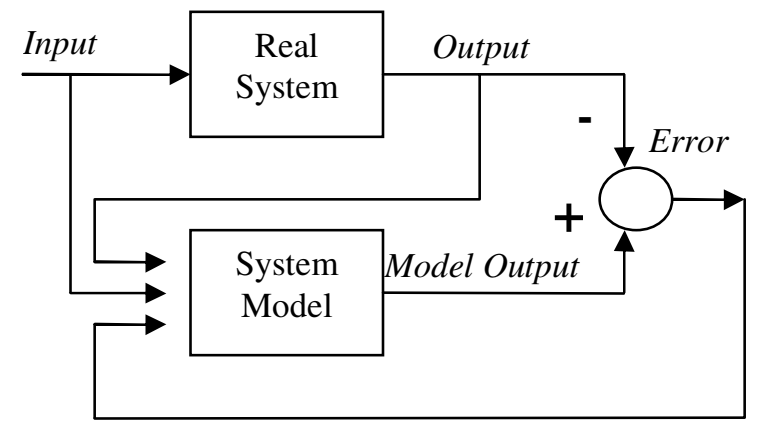

Figure 1. Prediction Error Model Block Diagram

The least square error for each sequence approximation is

$$
E(k)=\frac{1}{2}(\hat{y}(k)-y(k))^{2}
$$

This is the error function that is to be minimized. The variables are the parameters $a(j)$ and $b(i)$

Optimization Algorithms, such as Newton and steepest descent, can be used to minimize the above error. The gradient will be made of the following derivatives

$$
\frac{\partial E(k)}{\partial a(j)}=(\hat{y}(k)-y(k)) y(k-j)=e(k) y(k-j)(7)
$$

$$
\frac{\partial E(k)}{\partial b(i)}=(\hat{y}(k)-y(k)) x(k-i)=e(k) u(k-i)
$$

The steepest descent algorithm is used to get the following updates

$$
\begin{aligned}
& a(j)=a(j)-\alpha y(k-j) e(k) \\
& b(k)=b(k)-\alpha u(k-i) e(k)
\end{aligned}
$$

where $\alpha$ is the step length. The above equations are equal to the updates, $\theta(\kappa)$ in equation (4) when the matrix $Q(k)$ is set to be the identity matrix.

Once the parameters are identified, the Z-Transform of the $\operatorname{ARMA}(m, n)$ model is calculated to yield the estimated Transfer function of the model which is given by

$$
\begin{aligned}
& Z\left\{y(k)-\sum_{j=1}^{n} a(j) y(k-j)\right\}=Z\left\{\sum_{i=0}^{m} b(i) u(k-i)\right\} \\
& \Rightarrow H(z)=\frac{Y(z)}{U(z)}=\frac{b_{0}+b_{1} z^{-1}+\ldots+b_{M} z^{-m}}{a_{0}+a_{1} z^{-1}+\ldots+a_{N} z^{-n}}
\end{aligned}
$$

\section{MATHEMATICAL MODELING}

Gyroscope system consists of a DC motor in a solid aluminium frame. The motor is equipped with gearbox. The gearbox output drives external gears. The external gearbox drives gyro module. Figure 2 shows the gyroscope diagram

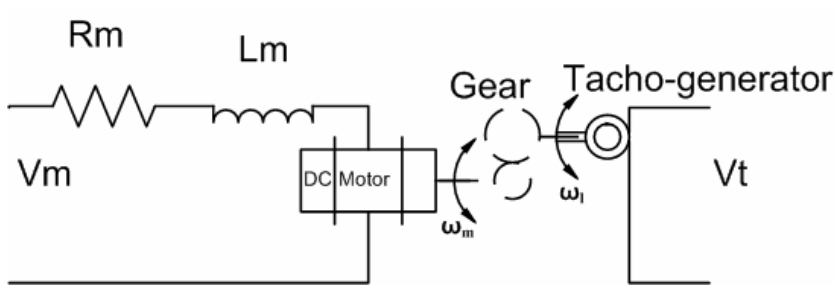

Figure 2. Gyroscope Diagram

The electrical equations can be derived using Kirchoff's voltage as follow

$V_{m}-R i-L \frac{d i}{d t}-V_{e m f}=0$

where $V_{m}$ is the armature input voltage, $i$ is the armature input current, $R$ is the armature resistance, $L$ is the armature inductance, and $V_{e m f}$ motor back-emf voltage

Since $L<<R$, we can disregard the motor inductance and therefore,

$i=\left(V_{m}-V_{e m f}\right) / R$ 
Back emf created by the motor is proportional to the motor shaft velocity $\omega_{\mathrm{m}}$

$$
i=\left(V_{m}-K_{m} \omega_{m}\right) / R
$$

where $\omega_{m}$ motor shaft angular velocity and $K_{m}$ is the motor mechanical constant.

The mechanical equations are found by applying Newton $2^{\text {nd }}$ law of motion to the motor shaft

$$
J_{m} \dot{\omega}_{m}=T_{m}-\left(T_{\text {load }} / \eta_{g} K_{g}\right)
$$

where $T_{m}$ is the torque generated by motor, $T_{\text {load }}$ is the torque applied at the load, $K_{g}$ is the gear ratio, and $\eta_{g}$ is the efficiency of the gearbox. Here the damping and spring effects are ignored motor gives

Applying the $2^{\text {nd }}$ law of motion at the load of the

$$
\begin{aligned}
& J_{\text {total }} \dot{\omega}_{\text {load }}=T_{\text {total }}-B_{\text {equ }} \omega_{\text {load }} \\
& J_{\text {total }}=\sqrt{J_{\text {load }}^{2}+J_{f}^{2} \cos \theta} \\
& \theta=\tan ^{-1} \frac{J_{\text {load }}}{J_{f}}
\end{aligned}
$$

where $B_{\text {equ }}$ is the viscous dumping coefficient, $J_{\text {total }}$ is the total moment of inertia, $J_{f}$ is the flywheel moment of inertia, $J_{\text {load }}$ is the load moment of inertia and $\omega_{\text {load }}$ is the load shaft angular velocity

$$
\begin{aligned}
& T_{\text {total }}=\sqrt{T_{\text {load }}^{2}+T_{f}^{2} \cos \phi} \\
& \phi=\tan ^{-1} \frac{T_{\text {load }}}{T_{f}}
\end{aligned}
$$

where $T_{f}$ torque generated by flywheel, $T_{\text {load }}$ is the torque applied at the load and $T_{\text {total }}$ is the total torque applied on the system

Using the gear ratio equations below

$$
\begin{aligned}
& \omega_{m}=K_{g} \omega_{\text {load }} \\
& T_{m}=\eta_{m} K_{t} i
\end{aligned}
$$

where $\eta_{m}$ is the motor efficiency and $K_{t}$ is the motor torque constant

In order to obtain the relationship between the input voltage $V_{m}$ and the angular velocity $\omega_{m}$ we need to manipulate equations (12) to (18). This relationship is highly nonlinear. This is evident by the cosine and square root functions in equations (17) and (19). Therefore, the relationship between the angular velocity output and the motor voltage input is non trivial.

\section{EXPERIMENTAL RESULTS}

The experimental results from the gyroscope are shown in figures 3 and 4. National Instruments NI6063E DAQ Card from with sampling rate up to $200 \mathrm{kHz}$ was used. The PC used was a Pentium 4 operating at $2.19 \mathrm{GHz}$. All results were obtained using Labview software.

Gyroscope system is a nonlinear system. We tested the recursive algorithm on the gyroscope to validate the system identification method used.

In the Gyroscope system experiment, the total duration time was set to $2.5 \mathrm{sec}$ and the sampling rate was set to 1000 samples per second for each channel. A total of three DAQ channels were used. The first channel was the input measured at the actual motor input signal measured at the motor rotor (after the drive circuit). The second channel was the measured motor speed input from the tachometer. The third channel was the impulse output to the drive circuit. As in the DC motor experiment, two software filters were used for the motor input and for tachometer output channel.

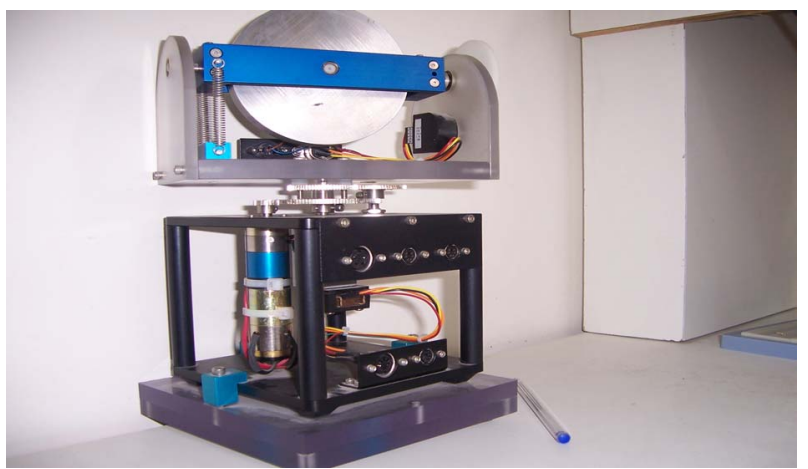

Figure 3. Gyroscope used in the experiment

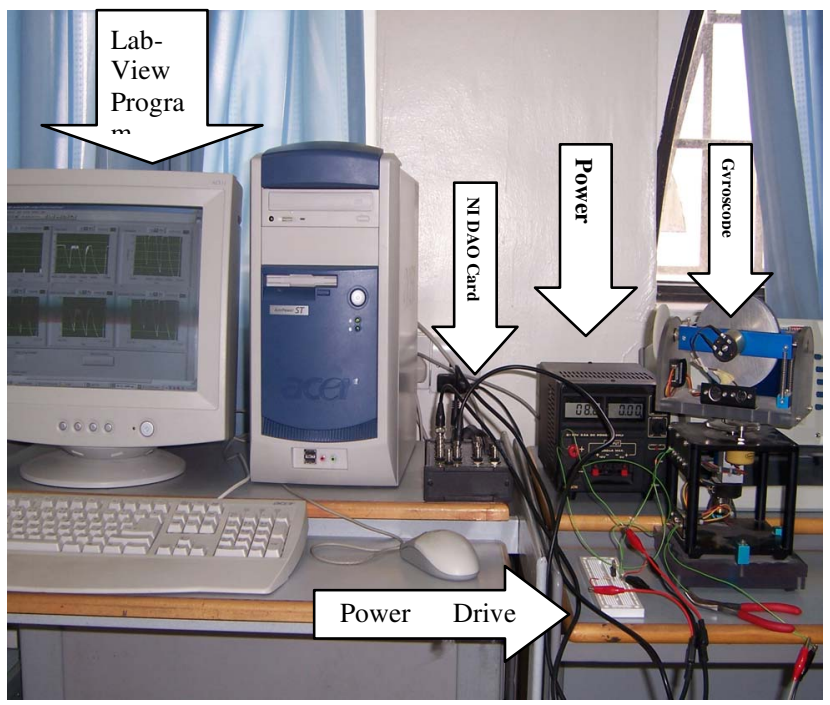

Figure 4. Gyroscope experiment setup

The impulse and response signals are shown in figure 5. 


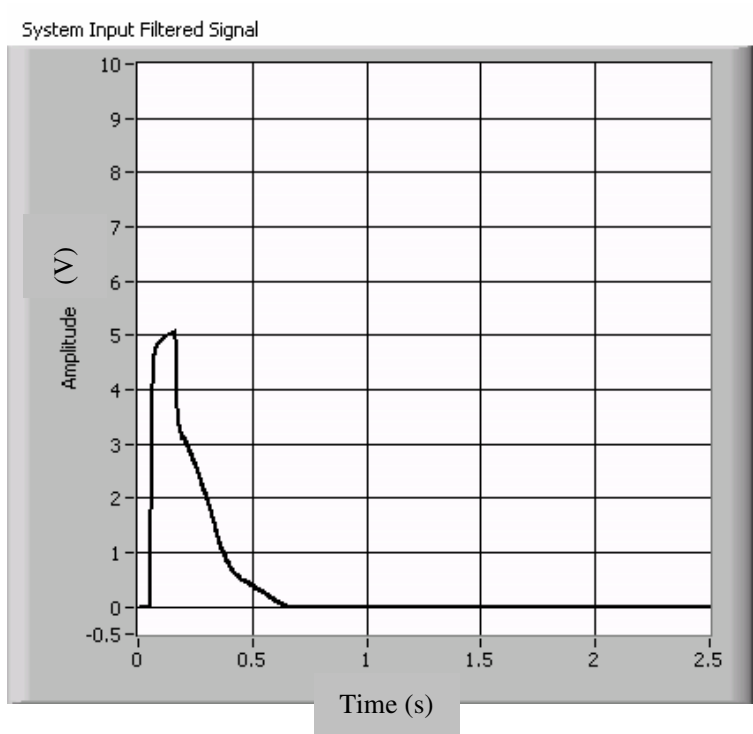

(a)

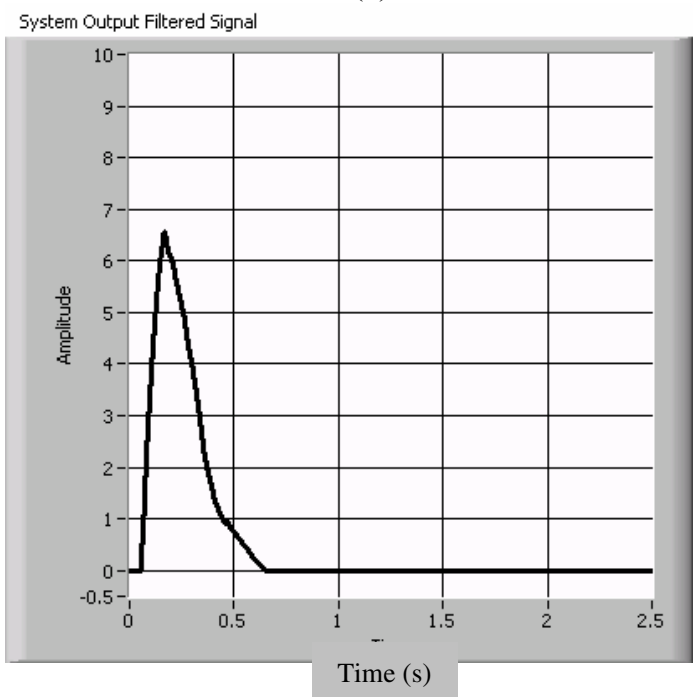

(b)

Figure 5 (a) Filtered input to the Gyroscope system,(b) Filtered output from tachometer

The transfer function was found to be

$$
H(z)=\frac{0.09-0.08 Z^{-1}-0.13 Z^{-2}+0.21 Z^{-3}-0.08 Z^{-4}}{1-2.20 Z^{-1}+1.55 Z^{-2}-0.45 Z^{-3}+0.10 Z^{-4}}
$$

It is important to note that this is not a unique transfer function, since other runs might produce different result.

Another impulse was given the physical system. The response of the identified transfer function was compared to the original physical system. These results are shown in figure 6 .

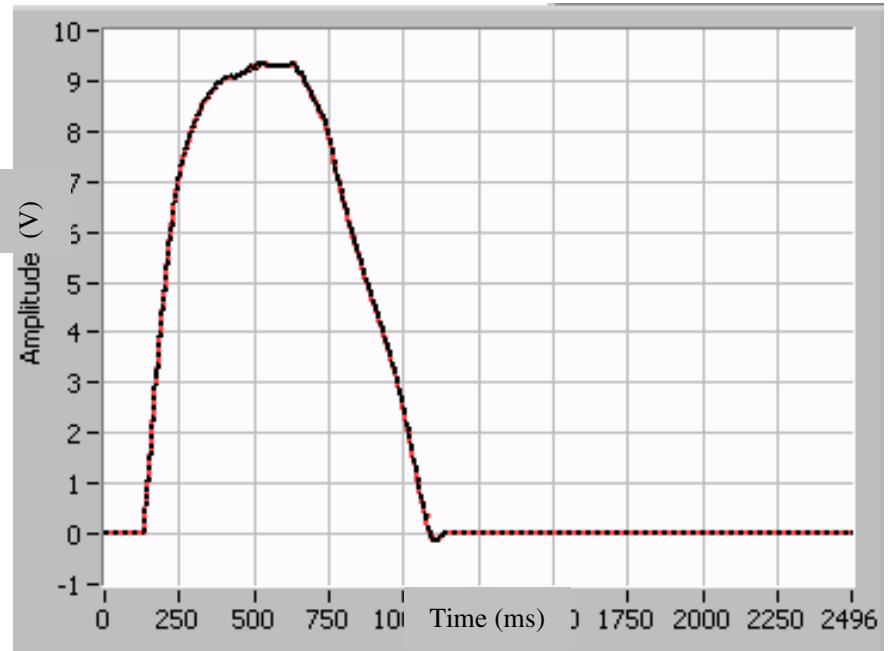

Figure 6. Identified output (dot line) vs. physical output (solid line)

In order to get better insight, figure 7 displays the error between the identified and the physical system. The mean square error was 0.0032

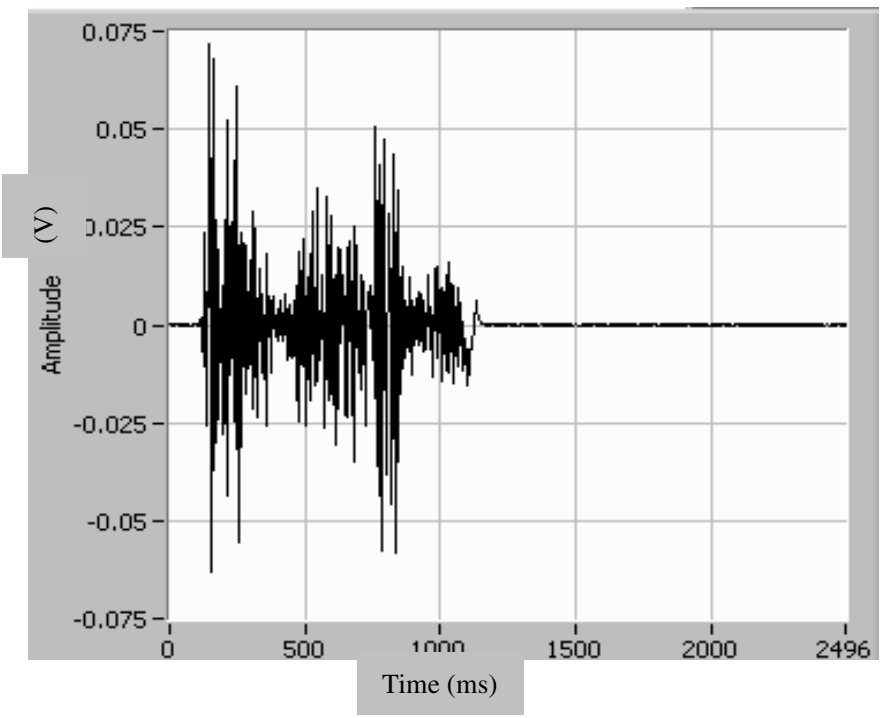

Figure 7. Error between the identified and the physical system

Finally noise was added noise to the system. The results are shown in figures 8 and 9 . The mean square error was 0.001 . 


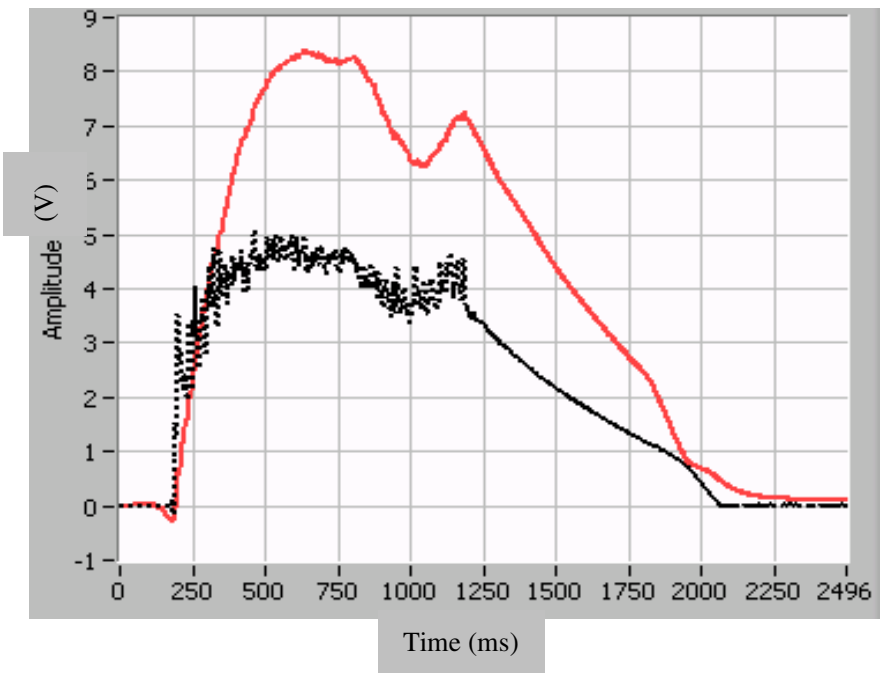

Figure 8. Output signal (solid line), input signal (dot line) with noise as applied to the gyroscope system

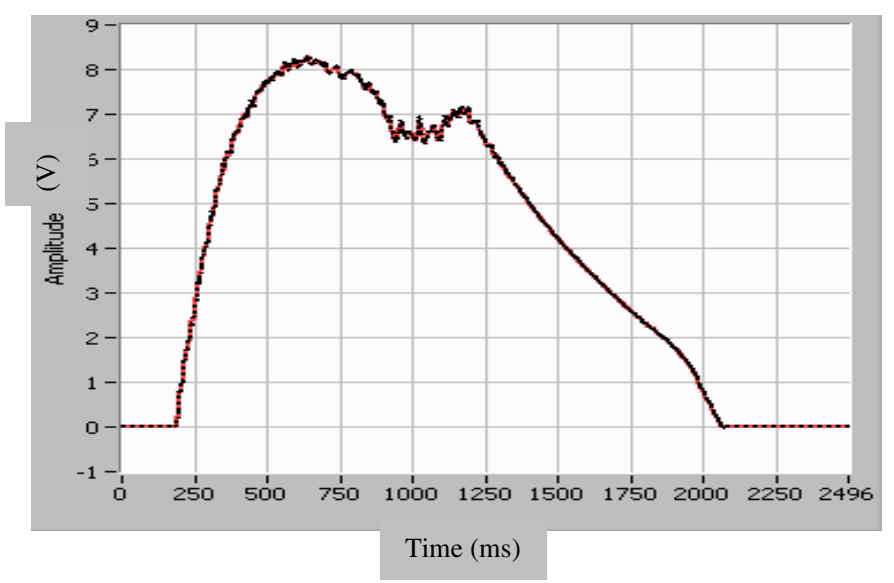

Figure 9. Output from identified transfer function (dot line) vs. physical output (solid line)

\section{CONCLUSIONS}

Recursive identification algorithm was used to identify a gyroscope system. The algorithm used a recursive least squares (RLS) algorithm to identify the ARMA parameters of the transfer function. The input-output patterns used were the impulse (excitation voltage) and impulse response (angular speed) to the gyroscope motor. Experimental results using NI DAQ with Labview were presented. All results showed that the proposed algorithm gave an accurate response of the identified systems.

\section{REFERENCES}

[1] Soderstrom and Stoica, System Identification, Prentice Hall 1989

[2] T. Kara and I. Eker, "Nonlinear modeling and identification of a DC motor for bidirectional operation with real time experiments". Energy
Conversion and Management 45 (2004) 1087-1106

[3] Ilyas Eker, "Open-loop and closed-loop experimental on-line identification of a three-mass electromechanical system". Mechatronics 14 (2004), 549-565

[4] Tutunji, "DC motor identification using impulse response data", EUROCON2005 Serbia and Montenegro, Belgrade Nov 22-24, 2005. pp 17341736

[5] B. Angerer, Hintz, and Shhroder, "Online identification of a nonlinear mechatronic system", Control Engineering Practice 12 (2004), 1465-1478

[6] Y. Koubaa. "Recursive identification of induction motor parameters", Simulation Modeling Practice and Theory 12 (2004) 363-381

[7] Yan, Lee, and Yen. "Theory and Application of a combined self-tuning adaptive control and crosscoupling control in a retrofit milling machine", Mechatronics (2004) Article in Press

[8] Ostring, Gunnarson, and Norloff. "Closed-loop identification of an industrial robot containing flexibilities", Control Engineering Practice 11 (2003) 291-300

[9] Johansson, Robertsson, Nilsson, and Verhaegen . "State-space system identification of robot manipulator dynamics", Mechatronics 10 (2001) 403-418

[10] Eyad Turki, System Identification of DC Motor using ARMA model. JUST (Jordan University of Science and Technology), MS Thesis 2006 